Recepción: 20 / 03 / 2019

Aceptación: 21 / 04 / 2019

Publicación: 05 / 05 / 2019

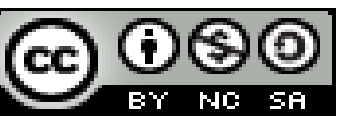

Ciencias de la salud

\title{
La gestión turística sostenible del patrimonio natural del Ecuador como herramienta para su conservación y desarrollo.
}

Sustainable tourism management of the natural heritage of the Ecuador as a tool for conservation and development

Gestão de turismo sustentável do património natural do Equador como uma ferramenta para conservação e desenvolvimento

Marcela Alexandra Galarza-Torres ${ }^{\mathrm{I}}$ alexandra.galarza@ucuenca.edu.ec

Correspondencia: alexandra.galarza@ucuenca.edu.ec

\footnotetext{
I. Máster en Dirección y Gestión Turística, Licenciado En Turismo, Facultad de Ciencias de la Hospitalidad,
} Universidad de Cuenca, Cuenca, Ecuador. 


\title{
Resumen
}

El auge que el ecoturismo ha alcanzado en los últimos años en el Ecuador, ha ocasionado que gran parte de las áreas protegidas acojan a miles de turistas nacionales y extranjeros quienes, motivados por la biodiversidad existente en ellas, buscan experiencias más cercanas con la biodiversidad y su cultura. En la actualidad, la administración del patrimonio natural recae en una institución gubernamental, cuya labor se esboza en el control y supervisión de los territorios declarados como protegidos, siendo la sostenibilidad un área todavía inexplorada. Este hecho puede generar impactos negativos considerables sobre el territorio y sobre la imagen de estos espacios considerados como destinos turísticos de naturaleza de gran relevancia en el país y sobre su capacidad como factor de desarrollo regional y rural. Desde esta perspectiva el objeto investigativo del presente trabajo fue analizar las distintas estrategias que se pueden adoptar desde la administración del patrimonio natural, para alcanzar una gestión turística sostenible que conlleve una práctica de un turismo más sostenible y responsable con el patrimonio natural del Ecuador, que a su vez permita generar mayor desarrollo para las comunidades loca-les. Para lograr el objetivo trazado, se realizó un estudio cualitativo de alcance descriptivo, donde la recolección de la información se logró obtener a través de la aplicación del método Delphi y con la aplicación de un cuestionario dirigido a los directores/guardaparques de tres áreas protegidas del Azuay.

Palabras claves: Gestión sostenible; ecoturismo; conservación; patrimonio natural.

\begin{abstract}
The boom that ecotourism has reached in recent years in Ecuador has caused many of the pro-tected areas to receive thousands of domestic and foreign tourists; those motivated by the existing biodiversity in them seek closer experiences with biodiversity and its culture. Howev-er, currently the administration of the natural heritage falls on a governmental institution, whose work is outlined in the control and supervision of the territories declared as protected, with sustainability being an area still unexplored. This fact can generate considerable negative impacts on the territory and on the image of these spaces considered as touristic destinations of a highly relevant nature in the country and on their capacity as a factor of regional and rural development. From this perspective the investigative object of the present work was to ana-lyze the different strategies that can be adopted from the
\end{abstract}


administration of the natural herit-age, to achieve a sustainable tourism management that entails a practice of a more sustainable and responsible tourism with the natural patrimony. of Ecuador, which in turn allows for greater development for local communities. In order to achieve the outlined objective, a quali-tative study of descriptive scope was carried out, where the collection of the information was obtained through the application of the Delphi method and with the application of a question-naire addressed to the directors / park rangers of three protected areas of the Azuay.

Keys words: Sustainable management; ecotourism; conservation; natural heritage.

\section{Resumo}

O boom que ecoturismo tem alcançado nos últimos anos no Equador, tem causado que acei-tam grande parte das áreas protegidas para milhares de turistas e estrangeiros; aqueles motiva-dos pela biodiversidade existente neles buscando experiências mais de perto com a biodiversi-dade e sua cultura. No entanto, atualmente a gestão do património natural é uma instituição governamental, cujo trabalho é descrito no controle e supervisão dos territórios declarado co-mo protegido, sendo a sustentabilidade uma área ainda inexplorada. Este fato pode gerar im-pactos negativos consideráveis no território e na imagem dessas áreas consideradas como des-tinos turísticos na natureza de grande relevância no país e na sua capacidade como factor de desenvolvimento regional e rural. Nesta perspectiva o objeto de pesquisa deste trabalho foi analisar as diferentes estratégias que podem ser adotadas pela gestão do património natural, para alcançar uma gestão turística sustentável envolvendo uma prática de turismo mais susten-tável e responsável com o património natural do Equador, que permite maior desenvolvimento para as comunidades locais. Para alcançar o mapeamento foi realizado um estudo qualitativo de escopo descritivo, onde a coleta de informações é alcançada através da aplicação do méto-do Delphi e da aplicação de um questionário dirigida a diretores/Rangers três áreas protegidas de Azuay.

Palavras chaves: Ecoturismo, conservação; manejo sustentável; património natural.

\section{Introducción}

El desarrollo del ecoturismo como actividad turística a nivel internacional crece a tasas muy superiores (entre un $10 \%$ y un 30\% anual) a la media de crecimiento de la actividad turística en general (4,3\% 
anual) según estadísticas del World Resources Institute (Wearing y Neil, 2000), siendo previsible que se mantenga esta tendencia en los próximos años.

El Ecuador no ha sido ajeno a este crecimiento, pues ha ido incrementándose considerablemente. Según datos del Ministerio de Ambiente del Ecuador (MAE) las áreas protegidas ecuatorianas han recibido 2 millones de visitas en el año 2015, de los cuales el 68\% de los turistas extranjeros que visitaron el país manifestaron que su principal motivación son las áreas protegidas y el ecoturismo; esta realidad obedece a la creciente conciencia y valoración del turista sobre los espacios naturales protegidos, así como también influye la promoción turística que la nación ha ido gestando en los últimos años en materia de turismo de naturaleza.

Actualmente, las áreas protegidas se encuentran gestionadas por el Ministerio del Ambiente a través del Sistema Nacional de Áreas Protegidas del Ecuador el cual abarca el 33\% de la superficie del territorio nacional MAE (2014). Este sistema alberga una gran variedad de ecosistemas y una biodiversidad muy importante que refleja la riqueza natural que posee el Ecuador.

Estos espacios naturales se han convertido en la actualidad en un destino clave y vital para la práctica del turismo en el Ecuador, sin embargo es prioritario contemplar políticas para su gestión sostenible, siempre y cuando pueda convertirse en un instrumento efectivo para la conservación de la naturaleza pero a su vez transformándose en una actividad productiva, considerando los requisitos necesarios para su gestión como la infraestructura y el talento humano que pueda asegurar su sostenibilidad a largo plazo, respetando los derechos de las poblaciones locales.

En este sentido, el Parque Nacional Cajas contempla dentro de su plan de manejo, estrategias encaminadas a la práctica de un turismo más responsable, no únicamente enfocados en el cuidado y conservación de la naturaleza, sino también en las comunidades que cohabitan este sitio maravilloso que además ostenta el título de Reserva de la Biósfera declarada por la UNESCO.

\section{Importancia del problema}

Las áreas protegidas generan una gran cantidad de beneficios, especialmente de carácter ambiental como son la conservación de los recursos hídricos, el secuestro de carbono y la conservación de biodiversidad, el paisaje también ha sido uno de los beneficios ambientales sobre los que se han 
desarrollado esquemas de Pagos por Servicios Ambientales (PSA). Las Áreas Protegidas atraen visitantes por su belleza escénica, riqueza natural y cultural.

Ecuador posee 58 áreas naturales protegidas, de las cuales el 33\% fueron creados en los últimos 9 años, hecho que evidencia una creciente consolidación referente a la importancia de la conservación de la biodiversidad terrestre y marina del país. Así también, entre 2012 y 2015 el Estado ecuatoriano invirtió alrededor de 51,3 millones de dólares en infraestructura turística MAE (2014). Entre los programas que se manejaron en ese período en las áreas protegidas están la administración y planificación, el control y vigilancia, la comunicación, educación y participación ambiental, el uso público y turismo y manejo de biodiversidad, no existe referente alguno sobre la gestión sostenible del patrimonio natural o de éste hacia el ecoturismo.

Sin embargo, el Sistema Nacional de Áreas Protegidas del Ecuador conforma un conjunto territorial no homogéneo ya que se presentan las características físicas, geológicas o ambientales, y por otro lado, los niveles de desarrollo económico de su entorno (especialmente patente, por ejemplo, en las diferencias entre las áreas naturales de la sierra ecuatoriana frente a las áreas de zonas litorales).

Igualmente, es importante señalar que no todas las áreas protegidas legalmente declaradas como tal en el país se le han otorgado el título con la finalidad de estimular estrategias de desarrollo sostenible; entre ellas la actividad turística ya que en Ecuador existen territorios cuya declaración responde exclusivamente a la preservación de zonas de especial fragilidad, como lo es Yasuní, Sumaco, LLanganates, Mazán, entre otros y resaltando la existencia de zonas de conflicto por uso de las tierras que alcanza en ciertos casos, niveles preocupantes de dificulta para la implementación de estas estrategias.

"En lo referente al nivel de desarrollo turístico en espacios protegidos naturales del país este ha ido incrementándose evidentemente en áreas como el Parque Nacional Galápagos que en el año 2017 recibió 241.800 turistas, reflejando un incremento del 11\% frente a 2016". (Informe Anual de Estadísticas Turísticas- Ministerio de Turismo, 2017). "El Parque Nacional Cotopaxi por su parte bordeó los 22.000 turistas, y el Parque Nacional Yasuní, que registró en 20176.500 turistas" (Diario el Telégrafo, 2017), también Cotacachi Cayapas que en el mismo año recibió a más de 240.000 turistas y el Área Nacional de Recreación el Boliche cuya intensidad de uso turístico, especialización turística, 
la oferta de infraestructuras, su equipamientos o actividades de uso público las hacen un referente turístico mucho mayor frente a otras áreas cuyo desarrollo todavía es muy incipiente.

Para Calvete (2007) el principio de permanencia se sustenta en la idea de que la transformación en los sistemas complejos es inevitable, planteando un cambio de enfoque bastante importante para las personas que tienen una tendencia a pensar en la permanencia de las cosas y por lo tanto realizan acciones pensando que nunca van a cambiar o que son un "derecho adquirido".

El principio de la magnitud de la transformación sustenta que el proceso transformador depende de las condiciones previas en las que se encontraba el sistema, bajo este concepto se diría como ejemplo que la magnitud del potencial para enfermarse no depende de la virulencia del atacante sino de la salud de la víctima. Y el principio de las condiciones esenciales, bajo esta cosmovisión se entiende que la sustentabilidad no habla sólo de la supervivencia de una especie, sino de las condiciones esenciales que tienen que darse para que esa especie pueda crecer y desarrollarse indefinidamente y se debe partir de la base fundamental e indiscutible de que nada puede existir aisladamente, así como tampoco nada puede evolucionar aisladamente.

Desde la perspectiva dimensional de la sustentabilidad se describen diversas dimensiones, las de mayor tradición han sido la social, la económica y la ambiental. Pero hoy en día, diferentes autores dependiendo de su disciplina y sector hablan de otras dimensiones, por ejemplo: la académica, espiritual, arquitectónica, política, paisajística, cultural, entre otras, mismas que cobran importancia en función del contexto que se tiene, y el rumbo que se le quiere dar a la sustentabilidad (Gutiérrez \& Martínez, 2009).

Es importante por tanto dotar a estos espacios protegidos de instrumentos que permitan garantizar la calidad de la visita turística, que por un lado permita obtener la satisfacción del usuario, en cuanto de conservación de los recursos naturales, que son la principal motivación turística de estos espacios por lo que aunque sólo fuese por una reacción puramente mercantilista, la conservación de estos valores debería ser el primer aspecto a considerar por parte de quienes se benefician de su desarrollo turístico. Pulido (2004). 
En consecuencia, es necesario definir una política turística que garantice una gestión sostenible de estos espacios, a la vez que favorezca su posicionamiento competitivo en los mercados, para convertirlos en auténticos destinos turísticos.

La dimensión ecológica establece la sostenibilidad en términos ecológicos, supone que la economía sea circular, que se produzca un cierre de los ciclos, tratando de imitar a la naturaleza. Es decir, "hay que diseñar sistemas productivos que sean capaces de utilizar únicamente recursos y energías renovables, y no producir residuos ya que éstos vuelven a la naturaleza" (Artaraz, 2002). Esta dimensión busca que el desarrollo sea compatible con el mantenimiento de los procesos ecológicos esenciales, la diversidad biológica y los recursos naturales. "También aparece como un criterio normativo para la reconstrucción del orden económico, como una condición para la sobrevivencia humana y un soporte para lograr un desarrollo durable" (Leff, 2002).

\section{Metodología}

La investigación realizada en el presente estudio tiene enfoque cualitativo de alcance descriptivo, para ello se emplearon varios estudios de caso de países como España, Argentina y Ecuador cuya trayectoria en el manejo de estrategias de gestión para un turismo sostenible, han marcado una trayectoria relevante. El estudio permitió establecer cuáles son las herramientas necesarias para un manejo efectivo del turismo sostenible en áreas naturales protegidas, integrando en este proceso a las comunidades anfitrionas cuya misión se ha visto desplegada a un papel secundario en este proceso.

Esta selección de estudios de caso, permitió establecer una profunda reflexión sobre cuál es el camino que deben seguir las áreas protegidas en función de su especialización turística, considerando para ello sus particularidades y componentes naturales, así como su problemática y relaciones exógenas con el entorno.

Por otra parte, se han utilizado bases de datos, construidas expresamente a partir de dos técnicas aplicadas también a dos grupos de expertos con características muy diferentes. La primera es el resultado de un estudio Delphi (cuadro 1) realizado con expertos regionales y nacionales, ampliamente conocedores de los distintos aspectos que caracterizan a los procesos de desarrollo turístico en las áreas protegidas de la provincia del Azuay y, en la mayoría de los casos, también en el resto del país. El objetivo fue obtener una visión externa y presumiblemente objetiva sobre las características de 
estos procesos, los principales déficit y retos a los que se enfrentan y las posibles políticas a desarrollar para hacer frente con garantía de éxito a esta situación.

\section{Cuadro 1.}

Ficha técnica del método Delphi

\begin{tabular}{ll}
\hline & Expertos provinciales en turismo de \\
Ámbito de estudio & naturaleza, conocedores de los procesos de \\
& desarrollo de las áreas naturales \\
protegidas. & \\
\hline Instrumentos de recolección de & 6 cuestionarios en la primera ronda \\
información aplicados & 4 cuestionarios en la segunda ronda \\
\hline Fecha del trabajo empírico & Inicio: diciembre 2017 \\
\hline Método empleado & Fin: julio 2018 \\
\hline
\end{tabular}

Fuente: Elaboración propia

La segunda fuente de información procede de un cuestionario dirigido a los directores/guardaparques de tres áreas protegidas del Azuay (cuadro 2), que buscaba conocer la percepción de los técnicos que disponen de mayor capacidad de decisión en la gestión de estos espacios, además de otros aspectos relativos a la actual gestión de los mismos, relacionadas con su uso turístico-recreativo. Además, se quiso conocer su opinión sobre la posibilidad de introducir la figura del gestor turístico, es decir, si en general, existe un ambiente propicio receptivo por parte de aquellos a la introducción de planteamientos, técnicas y profesionales dedicados a la gestión turística del parque natural objeto de su competencia, o si, por el contrario, se trata de un ambiente hostil, contrario a cualquier actuación que implique el reconocimiento del creciente interés turístico y recreativo de estos espacios. 


\section{Cuadro 2.}

Ficha técnica del cuestionario realizado a directores/guardaparques de áreas protegidas del Azuay

\begin{tabular}{ll}
\hline Universo de medida y ámbito de estudio & $\begin{array}{l}\text { Directores/guardaparques de } 5 \text { áreas } \\
\text { protegidas del Azuay. }\end{array}$ \\
\hline Personas entrevistadas & 5 \\
\hline Fecha del trabajo empírico & 12 de mayo de 2018 \\
& 22 de junio de 2018 \\
\hline Instrumento de recolección de & Cuestionario estructurado enviado por \\
información & correo electrónico \\
\hline Programa informático empleado & SPSS para Mac, versión 10.0 \\
\hline
\end{tabular}

Fuente: Elaboración propia

Además, se introdujeron algunas preguntas idénticas en la primera ronda del Delphi. y en el cuestionario realizado a los directores/guardaparques a fin de valorar el grado de coincidencia o de divergencia entre ambos grupos de encuestados (unos con una visión externa, quizás más asociada a la investigación y a la planificación, $\mathrm{y}$, a priori, más propensa a los procesos de desarrollo turístico; y los otros con una visión, también a priori, más subjetiva, de carácter ambientalista, incluso proteccionista, ligada a la gestión del día a día, a la toma real de decisiones que afectan al devenir del área protegida) respecto a determinados temas que se consideraban de interés para la propuesta final.

Una vez dispuesta la correspondiente base de datos, el trabajo empírico se desarrolló utilizando las siguientes variables estadísticas: media aritmética, mediana, moda y desviación típica

Turismo sostenible una alternativa viable para el desarrollo económico de las áreas protegidas 
Es la modalidad turística ejercida por personas naturales, jurídicas o comunidades legalmente reconocidas, previamente calificadas para tal efecto, a través de una serie determinada de actividades turísticas en áreas naturales, que correspondan o no al Sistema Nacional de Áreas Protegidas, con el objeto de conocer la cultura de las comunidades locales afincadas en ellas y/o la historia natural del entorno. Dichas actividades se ejercen con las precauciones necesarias para no alterar la integridad de los ecosistemas ni la cultura local y que generan oportunidades económicas que permiten la conservación de dichas áreas y el desarrollo de las comunidades locales, a través de un compromiso compartido entre ellas, las personas naturales o jurídicas privadas involucradas, los visitantes y el Estado.

Las áreas naturales protegidas representan el espacio más idóneo para fomentar prácticas de turismo de naturaleza sustentable; además tiene un gran valor como referentes de calidad ambiental al haber sido declaradas como protegidas y promueven la concientización de los visitantes sobre la importancia de la conservación (López, 2012).

Resulta por tanto importante la promoción del turismo sostenible en áreas protegidas, pues comprende una gran oportunidad no solamente por la posibilidad de conservar los recursos naturales del país, sino también porque la afluencia de turistas que pueden realizar pago por entradas aporta al financiamiento necesario para su gestión, aunque este monto es marginal dentro de las necesidades de recursos para mantener al sistema de manera integral. Los paisajes naturales son el principal elemento para la promoción de las regiones donde se desarrollan actividades turísticas, con la finalidad de crear la imagen de un destino para promocionarlo y venderlo en el mercado mundial (Aguilar, Palafox, \& Anaya, 2015).

El turismo en áreas protegidas es una actividad que genera beneficios para la conservación, la actividad turística y las comunidades locales. "Sin embargo, actualmente también constituye una amenaza a la conservación de los mismos sitios generadores de estos beneficios. Esta amenaza se debe principalmente a la falta de capacidad de manejo turístico del sistema" (Rodríguez, 2008). "La demanda turística mundial va tomando conciencia de la importancia de la conservación de los recursos patrimoniales, paralelamente crece su interés por el ecoturismo y el turismo cultural que busca vivencias auténticas, experiencias únicas e inolvidables mientras respeta el entorno aprendiendo de él”. (MAE, MINTUR, USAID, 2009). 
Comunidad vs. Turismo sostenible.

Según la Organización Mundial de Turismo [OMT] (2007) “el turismo se define como un fenómeno social, cultural y económico relacionado con el movimiento de las personas a lugares que se encuentran fuera de su lugar de residencia habitual por motivos personales o de negocios/profesionales". Estas personas se denominan visitantes y su desplazamiento implica un gasto turístico. Otra conceptualización indica que el turismo es el conjunto de actividades realizadas por las personas durante sus viajes y permanencias en lugares distintos al de su entorno habitual, por un período de tiempo consecutivo inferior a un año, por motivos diferentes al de ejercer una actividad remunerada en el lugar visitado.

Según la Federación de Parques Nacionales y Naturales, el Turismo Sostenible lo constituyen "todas las formas de desarrollo turístico, gestión, y actividad que mantienen la integridad ambiental, social y económica, así como el bienestar de los recursos naturales y culturales a perpetuidad” (Aragón, 2005).

En 1993, en la Conferencia Euromediterránea sobre Turismo y Desarrollo Sostenible, la Organización Mundial de Turismo (OMT) definió "el Turismo Sostenible como aquel que atiende a las necesidades de los turistas actuales y de las regiones receptoras y al mismo tiempo protege y fomenta las oportunidades para el futuro". Se concibe como una vía hacia la gestión de todos los recursos de forma que puedan satisfacerse las necesidades económicas, sociales y estéticas, respetando al mismo tiempo la integridad cultural, los procesos ecológicos esenciales, la diversidad biológica y los sistemas que sostienen la vida.

El turismo, genera directa e indirectamente un aumento de la actividad económica en los lugares visitados (y más allá de los mismos), fundamentalmente debido a la demanda de bienes y servicios que deben producirse y prestarse. En el análisis económico del turismo, se debe distinguir entre la 'contribución económica' del turismo que se refiere al efecto directo del turismo y que se puede medir, y el 'impacto económico' del turismo que es un concepto mucho más amplio que encapsula los efectos directos, indirectos e inducidos del turismo y que debe ser estimado mediante la aplicación de modelos. "En general hay en Ecuador tres líneas de productos claves: Ecoturismo-Turismo de Naturaleza, Turismo Cultural y Turismo de Deportes-Aventura, que configuran el mayor volumen de la oferta" (PLANDETUR, 2007). 
La política turística como herramienta para la gestión sostenible de espacios protegidos.

Monfort (2000), señala que "la política turística conserva una perspectiva de horizontalidad, en la medida en que está claramente influída por el carácter horizontal de las políticas instrumentales, que, en última instancia, condicionan sus resultados". A ello contribuyen, además, las peculiares características de la actividad turística, que la hacen especialmente receptiva a numerosas políticas de este tipo. Y, por otro lado, estas especiales características del turismo, y, sobre todo, la interrelaciónn de éste con otras actividades económicas, explican también la interferencia mutua entre la política turística y la mayor parte del resto de políticas sectoriales, que tienen una incidencia considerable en el desarrollo del turismo.

Por tanto resulta innegable una participación más activa del estado en el desarrollo turístico de las áreas protegidas, que permita por un lado instrumentar medidas que resalten aspectos o corrijan ciertas carencias (especialmente en la formación profesional de guías turísticos por ejemplo), que condicionan la capacidad competitiva del ecoturismo en el país; así también incidir con mayor intensidad en aquellos factores que contribuyen a aumentar la rentabilidad económica y social de la actividad turística en estos espacios protegidos, buscando mejorar la calidad de vida de la comunidad local, garantizando con ello un desarrollo turístico que respete el entorno y los valores culturales, naturales y sociales en el cual se desarrolla sino que a su vez promueva su mejora o regeneración. Pulido (2004).

\section{Las estrategias de gestión sostenible del turismo en espacios protegidos.}

En este apartado se pretende orientar sobre el empleo de una serie de estrategias, adaptadas tiempo atrás en los parques nacionales andaluces liderada por Pulido en 2004, y fue el fruto del trabajo realizado con la participación de los expertos Delphi y de los directores-conservadores, que tienen carácter holístico es decir, que abarcan todo el destino turístico y pretenden orientar la toma de decisiones de los agentes que participan en este proceso, así como, posteriormente, la elección de estrategias en el diseño del Plan de Gestión Turística de cada uno de las áreas naturales.

Estas estrategias también se han organizado en grandes grupos: estrategias de proceso, estrategias de gestión, estrategias de producto y estrategias transversales.

\section{Estrategias de proceso: Coordinación.}


Una de las principales falencias que se ha observado en el quehacer turístico en áreas protegidas, ha sido la descoordinación entre los distintos agentes que intervienen, en especial entre las administraciones ambientales y turísticas del país, los propios guardaparques o directores de área demuestran su falta de interés al valorar las relaciones con la gestión turística; a pesar de contar con presupuestos (en algunos casos) para el desarrollo del turismo, este interés va perdiendo altura conforme los cambios en las gubernamentales que rigen las carteras de estados involucradas.

\subsection{Estrategia de proceso: Participación.}

La participación activa de todos los agentes implicados en la gestión de un área protegida, es vital para garantizar una gestión turística sostenible eficiente; sin embargo, es fácil denotar que en muchos casos las poblaciones locales no tienen participación alguna en los planes operativos anuales o en los programas de desarrollo turístico, lo que demuestra un descontento y aparecen varias críticas al modelo de gestión que gran parte de las áreas protegidas implementan.

\subsection{Estrategia de proceso: La implicación empresarial.}

El desarrollo del turismo sostenible no recae únicamente al sector público, las empresas turísticas por ejemplo, que operan dentro de las áreas protegidas, tienen gran responsabilidad respecto de los impactos negativos del turismo, pero también, pueden involucrarse en la mayoría de estrategias de gestión sostenible del turismo, respecto de los cupos que se otorgan a operadores nacionales frente a los locales o al interés en fomentar la visitación de turistas nacionales frente a los extranjeros.

\section{Estrategias de gestión: Ordenación del territorio.}

El turismo es una actividad tremendamente devoradora del espacio y presenta una asombrosa capacidad de transformación del mismo, que puede, incluso, dilapidar el atractivo turístico de cualquier zona y comprometer su futuro Pulido (2004). "Por otro lado, la actividad turística implica el empleo y gestión de recursos progresivamente más escasos (agua, energía, suelo, paisaje, atractivos naturales,), que son compartidos, además, por otras actividades productivas, planteando el conocido dilema económico del uso alternativo de los recursos".

Garantizar la sostenibilidad del desarrollo turístico con respecto al territorio exige, en última instancia, la planificación conjunta de la actividad turística y el territorio y, por supuesto, un mayor control sobre 
el planeamiento urbanístico. Para ello se hace imperante el empleo de metodologías para zonificación de espacios acorde a su capacidad de regeneración o límites aceptables de productividad (cualquiera sea) dentro de estos espacios.

\subsection{Estrategia de gestión: conocimiento.}

Uno de los principales déficits detectados durante los últimos años, ha sido la falta de información sobre la mayoría (por no decir todos) de los aspectos relativos al desarrollo turístico en las áreas protegidas del país. Un caso a mostrar es que se carece de datos sobre la respuesta del territorio ante los impactos generados por el fenómeno turístico, sobre la incidencia real del turismo en la estructura socioeconómica y empresarial de los municipios que forman el área de influencia de las áreas naturales, sobre la demanda actual de uso turístico-recreativo (cuantificación, tipologías, expectativas, nivel de satisfacción, demandas e intereses), o sobre la demanda potencial (principales mercados, destinos competidores, etc.). Por tanto, es imperioso trabajar en un sistema de cuentas satélites que permita determinar cada uno de estos aspectos que buena falta le hacen a la gestión del ecoturismo en el Ecuador.

\subsection{Estrategia de gestión: La gestión de los visitantes.}

Una parte considerable de la frecuentación de las áreas protegidas naturales ecuatorianas se manifiesta en forma de uso recreativo (también llamado «ocio de proximidad», es decir, desplazamiento diario para la práctica de actividades de ocio en la naturaleza, pero sin pernoctación). En la actualidad, no hay diferencia entre el uso turístico y el recreativo, ni en la prestación de servicios ni en la gestión; todos son considerados visitantes que realizan actividades «turísticas» (difíciles de disociar de lo que se considera ocio o recreación) en el interior de un espacio natural protegido. Sin embargo, no todos tienen el mismo comportamiento, ni las mismas expectativas, ni manifiestan las mismas demandas, ni, por tanto, requieren la misma atención.

Por otro lado, lo que sí suele ser habitual es que todos terminen concentrándose en determinados lugares del área protegida (habitualmente, los más frecuentados), superando en determinados 
momentos su capacidad de acogida y poniendo en peligro, por tanto, la preservación de los recursos de esos lugares. Se trata, por tanto, de buscar alternativas encaminadas a posicionar nuevos destinos de naturaleza que promuevan la práctica responsable del turismo y que a su vez éste se constituya en una fuente sostenible de ingresos para las comunidades locales, por ejemplo, las amazónicas.

\section{Estrategias de producto: Promoción y comercialización.}

Si se habla en materia de promoción turística en Ecuador, Galápagos lleva la delantera, pues gracias a este destino el país se encuentra dentro de los destinos turísticos de naturaleza más visitados de Sudamérica, sin embargo, esta situación no constituye la realidad de las demás áreas protegidas del país, esto debido a una falta de una consolidación de una estrategia de comercialización integral del producto de naturaleza, dejando a la vista la dependencia que se mantiene como destino turístico a un espacio de gran fragilidad como lo es el archipiélago.

\section{Estrategias transversales: Capital humano.}

Una formación integral en materia turística que involucre al personal que labora dentro de las áreas protegidas, así como a las comunidades locales que intervienen en el quehacer turístico; para ello esta estrategia debe involucrar capacitaciones periódicas en tecnologías de la información para el ordenamiento territorial, atención y servicio al cliente, en cuanto a sostenibilidad y buenas prácticas ambientales.

\section{Estrategia de educación / sensibilización.}

Acorde a trabajos investigativos previos, los visitantes de las áreas protegidas tienen una escasa "cultura" ambiental, que se traduce en una sensibilización muy baja hacia la conservación de los recursos naturales y a un escaso interés por conocer y disfrutar el valor real de estos espacios; para ello los programas de educación ambiental tienen que convertirse en verdaderos escenarios que provean la sensibilización del visitante y llamando a una reflexión sobre el papel que cada uno desempeña frente a esta gestión.

\section{Resultados}


El análisis realizado a través del grupo de expertos Delphi y de los directores/guardaparques de las áreas protegidas de la provincia del Azuay, ha puesto de manifiesto la inexistencia de una política activa de gestión turística sostenible de estos territorios, que, como ellos mismos reconocen, está poniendo en peligro la preservación de estos espacios ante el constante aumento de flujos turísticos, avance de la frontera agrícola y ganadera en la región.

El trabajo aquí presentado ha permitido identificar la importancia de generar una política turística de las áreas protegidas del Azuay, o de los de cualquier otra provincia del país, como principal herramienta para una gestión turística sostenible ya que la situación desde el punto de vista de su gestión turística es prácticamente idéntica.

Los resultados obtenidos, evidencian que es posible definir e implantar una política de gestión turística activa y adaptable en espacios naturales protegidos, pero ello requiere, cambios profundos en las formas de actuar de la propia administración a la que compete su gestión, así como una nueva dinámica en la que primen los principios de cooperación, corresponsabilidad y participación.

Por otro lado, el diagnóstico ha resaltado, además, la existencia de importantes déficits de información respecto a la mayoría de los aspectos relacionados con el desarrollo turístico de estos territorios: incidencia en la estructura socioeconómica y empresarial, impactos sobre el medio ambiente, demanda actual y/o potencial de uso turístico-recreativo, entre otros. Asimismo, se han detectado serias carencias de comunicación entre los gestores de estos territorios y el resto de agentes implicados, especialmente los propios visitantes.

Además, se reconoce la existencia de una oferta turística dispersa y absolutamente desestructurada, que adolece de los problemas propios de un proceso de desarrollo emergente, acentuados en este caso por la profusión de conceptos y términos promocionales contradictorios, que sobredimensionan las expectativas de una demanda que termina crecientemente insatisfecha al no coincidir la imagen generada y transmitida con la realidad del desarrollo turístico de cada espacio.

Todo ello requiere de una intervención activa, de una política turística adecuada, que es la que se ha tratado de delimitar en este trabajo, pero también de un volumen de financiación adecuado al nivel de intervención requerido. A este respecto, se ha constatado la disconformidad de los visitantes de estos espacios a pagar por la mayor parte de los servicios recibidos en un parque natural y, por supuesto, su 
negativa a pagar entrada, en la medida en que se consideran bienes públicos de los que todos tienen derecho a disfrutar. Por tanto, en un principio, la autofinanciación es impensable en circunstancias como las actuales. Sin embargo, es evidente que el presupuesto público es incapaz de soportar el coste de una gestión turística activa de estos espacios, máxime en una provincia que cuenta con un número tan alto de espacios protegidos, muchos de los cuales disponen de una creciente demanda de uso turístico-recreativo. Es innegable, por tanto, que tarde o temprano deberá plantearse la posibilidad de obtención de ingresos por otras vías distintas del presupuesto público.

Por lo demás, esta situación se viene produciendo en un número cada vez mayor de sistemas nacionales de áreas protegidas en todo el mundo, existiendo ya una amplia gama de posibilidades y de experiencias de aplicación.

\section{Conclusiones}

La realidad que experimentan gran parte de las áreas protegidas en el país, denota la falta de una política activa que promueva la gestión sostenible del turismo en estos espacios, que está poniendo en peligro la conservación de los recursos frente a una creciente demanda de turistas que buscan satisfacer sus necesidades de ocio y recreación en entornos naturales altamente conservados.

El presente trabajo buscó orientar sobre el empleo de estrategias encaminadas a una gestión más sostenible de la actividad turística en espacios naturales protegidos. La realidad que experimentan gran parte de estas áreas, indica que es posible definir y desarrollar una política de gestión turística perfectamente aplicable y adaptable al patrimonio natural del Ecuador, sin embargo, ello también requiere de un cambio profundo en la responsabilidad que mantiene la administración pública frente a su gestión y a la implantación de principios de cooperación, corresponsabilidad y participación comunitaria.

La conclusión más representativa de este trabajo investigativo, resalta en la gran necesidad de contar con herramientas que efectivicen una gestión turística más sostenible de la actividad turística en áreas naturales protegidas, entre ellas, el contar con una política turística que permita que el área se inserte en su propio entorno natural y cultural, pues la demanda de espacios con fines de ocio y recreación cada vez son más demandados. 


\section{Referencias Bibliográficas}

Aguilar, A., Palafox, A., \& Anaya, J. (2015). El turismo y la transformación del paisaje natural, Madrid, España NÓESIS, 19-29.

Aragón, J. T. (2005). Jornadas de turismo sostenible. Aragón, España: Gobierno de Aragón.

Artaraz, M. (2002). Teoría de las 3 dimensiones de desarrollo sostenible. España.: Ecosistemas Revista de Ecología y Medio Ambiente.

Calvete, A. (2007). El concepto moderno de sustentabilidad. Santa Fe, Argentina: Centro de Altos Estudios Globales, Universidad Abierta Interamericana.

Consejería de Medio Ambiente, Sevilla, España (2004). El empleo y las actividades ecoturísticas en Andalucía: Recuperado de: http://www.juntadeandalucia.es/medioambiente/ documentos tecnicos/ecoturismo/introduccion.html

Gutiérrez, B., \& Martínez, M. (2009). Dimensiones de sustentabilidad en las instituciones de educación superior. Quito, Ecuador. Revista de la Educación Superior, 113-124.

Leff, E. (2002). Saber ambiental: Sustentabilidad, racionalidad, complejidad, poder. Madrid, España.: Siglo Veintiuno.

López, F. (2004). La gestión turística de los espacios naturales. En IX Congreso AECIT, Madrid, España.

López, M. Nulán. (2012). El capital social cognitivo para el desarrollo turístico sustentable en la reserva de la biósfera parque Atlántico Mar Chiquito, Argentina.

MAE - PSF. (2016). Áreas protegidas del Ecuador - Socio Estratégico para el Desarrollo, Quito: Manthra Comunicación. 
Marchena, M., et al. (1992). Ocio y turismo en los parques naturales andaluces. Dirección General de Turismo, Sevilla, España

Mill, R. CH. and Morrison, A. M. (1992). The tourism system: an introductory. Text, New Jersey, Estados Unidos: Prentice Hall.

Ministerio del ambiente. (2004). Cifras del ecoturismo en el Ecuador. Quito, Ecuador.

Monfort, V. M. (2000). La política turística: una aproximación. Cuadernos de Turismo, 6, pp. 7-27.

Organización Mundial del Turismo.(2007). Turismo: Panorama 2020. Volumen 7. Previsiones mundiales y perfiles de los segmentos de mercado. Organización Mundial del Turismo. Madrid, España.

PANE, MAE, MINTUR, USAID. (2009). Estrategia de mercadeo para el patrimonio de áreas naturales del Estado. Alianza ecuatoriana para el turismo sostenible. Quito, Ecuador.

PLANDETUR, E. E. (2007). PLANDETUR 2020, Quito, Ecuador: Europraxis.

Posso Yépez, M. (2011). Proyectos, Tesis y Marco Lógico. Quito, Ecuador: Noción.

Pulido, J. I. (2000). Ordenación, planificación y gestión del desarrollo turístico en espacios naturales protegidos: hacia un desarrollo turístico sostenible. Escuela Universitaria de Turismo, Universidad de Alcalá. Documento de Trabajo Serie A No 200002.

Pulido, J. I. (2003). Turismo de naturaleza y sostenibilidad a Distancia. Monográfico «Turismo y sostenibilidad», 21 (1), pp. 32-46.

Pulido, J.I. (2004). Elementos para orientar la formulación de una política turística sostenible en los parques naturales andaluces. Cuadernos de Turismo No 19. Andalucía, Sevilla, España. 
La gestión turística sostenible del patrimonio natural del Ecuador como herramienta para su conservación y desarrollo

Pulido, J. I. (2005). Criterios para una política turística sostenible en los parques naturales de Andalucía Consejería de Turismo, Comercio y Deporte, Junta de Andalucía. Sevilla, España.

Rainforest-Alliance. (2013). Turismo Sostenible en acción: cinco historias exitosas.

Wearing S. y Neil J. (2000). Ecoturismo: impacto, tendencias y posibilidades. Madrid, España: Síntesis. 